PROCEEDINGS OF THE

AMERICAN MATHEMATICAL SOCIETY

Volume 138, Number 4, April 2010, Pages 1525-1531

S 0002-9939(09)10217-4

Article electronically published on December 8, 2009

\title{
ON NONCONTRACTIBLE COMPACTA WITH TRIVIAL HOMOLOGY AND HOMOTOPY GROUPS
}

\author{
UMED H. KARIMOV AND DUŠAN REPOVŠ \\ (Communicated by Alexander N. Dranishnikov) \\ Dedicated to the memory of Professor Evgenij Grigor'evich Sklyarenko (1935-2009)
}

\begin{abstract}
We construct an example of a Peano continuum $X$ such that: (i) $X$ is a one-point compactification of a polyhedron; (ii) $X$ is weakly homotopy equivalent to a point (i.e. $\pi_{n}(X)$ is trivial for all $n \geq 0$ ); (iii) $X$ is noncontractible; and (iv) $X$ is homologically and cohomologically locally connected (i.e. $X$ is an HLC and $c l c$ space). We also prove that all classical homology groups (singular, Čech, and Borel-Moore), all classical cohomology groups (singular and Čech), and all finite-dimensional Hawaiian groups of $X$ are trivial.
\end{abstract}

\section{INTRODUCTION}

It is a fundamental fact of homotopy theory that the existence of a weak homotopy equivalence $f: K \rightarrow L$ between two $C W$-complexes $K$ and $L$ implies that $f$ is actually a homotopy equivalence $\left(K \simeq_{w} L \Longrightarrow K \simeq L\right)$. Therefore if a $C W$-complex $K$ has all homotopy groups trivial, then $K$ is necessarily contractible 15.

However, this is no longer true outside the class of $C W$-complexes, e.g. the Warsaw circle $W$ is an example of a planar noncontractible non-Peano continuum all of whose homotopy groups are trivial (cf. e.g. 11]). The failure of local connectivity of $W$ is crucial, since it is well-known that every planar simply connected Peano continuum must be contractible (cf. e.g. [11).

In our earlier paper [8] we constructed an example of a noncontractible Peano continuum with trivial homotopy groups. In the present paper we shall construct in some sense a sharper example, namely a noncontractible Peano continuum $X$ which is a one-point compactification of a polyhedron $P$, which is homologically locally connected (HLC space) and is weakly homotopy equivalent to a point $X \simeq_{w} *$.

Received by the editors November 25, 2008, and, in revised form, August 7, 2009, and September 18, 2009.

2010 Mathematics Subject Classification. Primary 54F15, 55N15; Secondary 54G20, 57M05.

Key words and phrases. Noncontractible compactum, weak homotopy equivalence, reduced complex $\widetilde{K}_{\mathcal{C}}$-theory, admissible spectrum, Peano continuum, infinite-dimensional Hawaiian earrings, Hawaiian group.

(C)2009 American Mathematical Society 
We shall also prove that all classical homology groups (singular, Čech, and Borel-Moore), all classical cohomology groups (singular and Čech) and all finitedimensional Hawaiian earring groups of this space $X$ are trivial. This answers our problem formulated in [8]. We shall also state some new open problems.

\section{Preliminaries}

We start by fixing some terminology and notation which will be used in the proof. All undefined terms can be found in 4, 6, 8, 9, 15.

For any topological space $Z$ with a base point $z_{0} \in Z$ the reduced suspension $S\left(Z, z_{0}\right)$ is defined by

$$
S\left(Z, z_{0}\right)=(Z \times I) /\left((Z \times\{0\}) \cup(Z \times\{1\}) \cup\left(z_{0} \times I\right)\right),
$$

where $I$ is the unit interval $I=[0,1] \subset \mathbb{R}$, and the unreduced suspension $S^{\prime}(Z)$ of $Z$ is defined by

$$
S^{\prime}(Z)=(Z \times I) /((Z \times\{0\}) \cup(Z \times\{1\})) .
$$

The reduced cone $C\left(Z, z_{0}\right)$ over $Z$ is defined by

$$
C\left(Z, z_{0}\right)=(Z \times I) /\left((Z \times\{1\}) \cup\left(z_{0} \times I\right)\right),
$$

and the unreduced cone $C^{\prime}(Z)$ over $Z$ is defined by

$$
C^{\prime}(Z)=(Z \times I) /(Z \times\{1\}) .
$$

The $n$-dimensional Hawaiian earring $(n=0,1,2, \ldots)$ is the following subspace of the Euclidean $(n+1)$-space $R^{n+1}$ :

$$
\mathcal{H}^{n}=\left\{\bar{x}=\left(x_{0}, x_{1}, \ldots x_{n}\right) \in \mathbb{R}^{n+1} \mid\left(x_{0}-1 / k\right)^{2}+\Sigma_{i=1}^{n} x_{i}^{2}=(1 / k)^{2}, k \in \mathbb{N}\right\} .
$$

In other words, $\mathcal{H}^{n}$ is a compact bouquet of a countable number of $n$-dimensional spheres $S_{k}^{n}$ of radius $1 / k$. The point $\theta=(0,0, \ldots)$ is the base point of $\mathcal{H}^{n}$. Obviously, $S\left(\mathcal{H}^{n}, \theta\right) \cong \mathcal{H}^{n+1}$ and $\pi_{n+1}\left(S\left(\mathcal{H}^{n}, \theta\right)\right)$ is an uncountable group, whereas $\pi_{n+1}\left(S^{\prime}\left(\mathcal{H}^{n}\right)\right)$ is a countable group.

A reduced complex $\widetilde{K}_{\mathcal{C}}^{*}$-theory is an extraordinary cohomology theory defined on the category of pointed compacta and homotopic mappings with respect to base points. For any compact pair of spaces $(X, A)$ with the base point $x_{0} \in A$, $\left(X, A, x_{0}\right)$, there exists the following long exact sequence (cf. e.g. [6. p. 55]):

$$
\cdots \rightarrow \widetilde{K}_{\mathcal{C}}^{n}(X / A) \rightarrow \widetilde{K}_{\mathcal{C}}^{n}(X) \rightarrow \widetilde{K}_{\mathcal{C}}^{n}(A) \rightarrow \widetilde{K}_{\mathcal{C}}^{n+1}(X / A) \rightarrow \cdots .
$$

We denote the homotopy classes of mappings with respect to the base point by $[$,$] . On the category of connected spaces, there exists a natural isomorphism of$ cofunctors, for some $C W$-complex $B U$ (cf. e.g. [9, Theorem 1.32]):

$$
\widetilde{K}_{\mathcal{C}}^{0}(X) \cong[X, B U]
$$

Every $C W$-complex is an absolute neighborhood retract (ANR). Therefore the functor $\widetilde{K}_{\mathcal{C}}^{0}$ is continuous; i.e. if $X_{i}$ are compact spaces and $X=\varliminf_{\longleftarrow} X_{i}$, then

$$
\widetilde{K}_{\mathcal{C}}^{0}(X) \cong \lim _{\longrightarrow} \widetilde{K}_{\mathcal{C}}^{0}\left(X_{i}\right)
$$




\section{The CONSTRUCTION OF EXAMPLES \\ AND THE PROOFS OF THE MAIN RESULTS}

Let $\mathcal{P}$ be an inverse sequence of finite $C W$-complexes $P_{i}$ :

$$
P_{0} \stackrel{f_{0}}{\longleftarrow} P_{1} \stackrel{f_{1}}{\longleftarrow} P_{2} \stackrel{f_{2}}{\longleftarrow} \cdots .
$$

Suppose that $P_{0}=\left\{p_{0}\right\}$ is a singleton and that all $P_{i}$ are regular finite $C W$ complexes, i.e. that they admit a finite polyhedral structure. Let $C\left(f_{0}, f_{1}, f_{2}, \ldots\right)$ be the infinite mapping cylinder of $\mathcal{P}$ (cf. e.g. [10, 12]) and let $\widetilde{\mathcal{P}}$ be its natural compactification by the inverse limit $\lim \mathcal{P}$.

Then the space $\widetilde{\mathcal{P}}$ is an absolute retract (AR) (cf. 10]). Let $P^{*}$ be the quotient space of $\widetilde{\mathcal{P}}$ by $\lim \mathcal{P}$. Obviously, the space $P^{*}$ is homeomorphic to the one-point compactification of the countable polyhedron $C\left(f_{0}, f_{1}, f_{2}, \ldots\right)$.

Let $C\left(f_{n}, f_{n+1}, \ldots, f_{m}\right)$ be the finite cylinder of mappings:

$$
P_{n} \stackrel{f_{n}}{\longleftarrow} P_{n+1} \stackrel{f_{n+1}}{\longleftarrow} \cdots P_{m} \stackrel{f_{m}}{\longleftarrow} P_{m+1} .
$$

Clearly, we may assume that

$$
P_{n} \cup P_{m+1} \subset C\left(f_{n}, f_{n+1}, \ldots, f_{m}\right) \subset C\left(f_{0}, f_{1}, f_{2}, \ldots\right) .
$$

We shall say that an inverse spectrum $\mathcal{P}$ is admissible if the following conditions are satisfied:

(1) every $P_{i}$ contains only one 0 -dimensional cell $p_{i}$ which is a base point and $f_{i}\left(p_{i+1}\right)=p_{i}$

(2) no $C W$-complex $P_{i}$ contains any cells of positive dimension less than $i$; and

(3) for $i \geq 0$, every homomorphism $\widetilde{K}_{\mathcal{C}}^{0}\left(f_{i}\right)$ is a nontrivial isomorphism.

Such admissible spectra do exist. For example, the inverse spectra constructed by Taylor [13] or the suspension of the spectra constructed by Kahn [7] are admissible spectra (in our sense).

Theorem 3.1. Let $\mathcal{P}$ be an admissible spectrum. Then the one-point compactification $P^{*}$ of the countable polyhedron $C\left(f_{0}, f_{1}, f_{2}, \ldots\right)$ has the following properties:

(i) $X$ is weakly homotopy equivalent to a point, $P^{*} \simeq_{w} *$ (i.e. $\pi_{n}(X)$ is trivial for all $n \geq 0)$;

(ii) $P^{*}$ is acyclic with respect to all classical homology groups (singular, BorelMoore, and (̌ech);

(iii) $P^{*}$ is acyclic with respect to all classical cohomology groups (singular and (̌ech);

(iv) $P^{*}$ is homologically locally connected (HLC);

(v) $P^{*}$ is cohomologically locally connected (clc); and

(vi) $P^{*}$ is a noncontractible Peano continuum.

Proof. By the sequence (1), we have the following natural exact sequence:

$$
\cdots \rightarrow \widetilde{K}_{\mathcal{C}}^{0}(\widetilde{\mathcal{P}}) \rightarrow \widetilde{K}_{\mathcal{C}}^{0}\left(\lim _{\longleftarrow} \mathcal{P}\right) \rightarrow \widetilde{K}_{\mathcal{C}}^{1}\left(P^{*}\right) \rightarrow \widetilde{K}_{\mathcal{C}}^{1}(\widetilde{\mathcal{P}}) \rightarrow \cdots
$$

As was mentioned before, $\widetilde{\mathcal{P}}$ is an absolute retract; therefore $\widetilde{K}_{\mathcal{C}}^{n}(\widetilde{\mathcal{P}})=0$. On the other hand, the group $\widetilde{K}_{\mathcal{C}}^{0}(\lim \mathcal{P})$ is nontrivial since the cofunctor $\widetilde{K}_{\mathcal{C}}^{0}$ is continuous and all homomorphisms $\widetilde{K}_{\mathcal{C}}^{0}\left(f_{i}\right)$ are nontrivial isomorphisms. It follows by exactness of the sequence (1) that the group $\widetilde{K}_{\mathcal{C}}^{1}\left(P^{*}\right)$ is also nontrivial and hence the space $P^{*}$ must be noncontractible, as asserted. 
Let us prove that all homotopy groups $\pi_{n \geq 1}\left(P^{*}\right)$ are trivial. Fix a number $n \in \mathbb{N}$. Let $m \in \mathbb{N}$ be any number such that $m>n$. Consider a relative $C W$-complex $\left(P^{*}, C\left(f_{m}, f_{m+1}, \ldots\right)^{*}\right)$ with the compactification point $*$ as the base point. Let $f$ be any mapping of the sphere $S^{n}$ with some base point $p t$ to $P^{*}$ i.e. we have a mapping $f$ of the relative $C W$-complex $\left(S^{n}, p t\right)$ to $\left(P^{*}, C\left(f_{m}, f_{m+1}, \ldots\right)^{*}\right)$.

By the Cellular Approximation Theorem (cf. e.g. [15]) the mapping $f$ is homotopic relative $p t$ to a cellular map

$$
g_{m}:\left(S^{n}, p t\right) \rightarrow\left(P^{*}, C\left(f_{m}, f_{m+1}, \ldots\right)^{*}\right) .
$$

Note that the space $P^{*}$ can be represented as the union of the following three spaces:

$$
P^{*}=C\left(f_{0}, f_{1}, \ldots, f_{m-1}\right) \cup C\left(f_{m}\right) \cup C\left(f_{m+1}, \ldots\right)^{*} .
$$

Observe that $C W$-complex $C\left(f_{m}\right)$ consists of two 0-dimensional cells, one 1dimensional cell and some cells of dimension larger than $n$, since $m>n$ by our choice of the number $m$. Since the mapping $g_{m}$ is a cellular mapping of the pairs it follows that the image of $g_{m}$ lies in the union of the $n$-dimensional skeleta of $P^{*}$ and $C\left(f_{m+1}, \ldots\right)^{*}$.

Since $C\left(f_{m}\right)$ contains only one 1-dimensional cell $e^{1}$ and cells of dimension larger than $n$, we have

$$
\operatorname{Im}\left(g_{m}\right) \subset C\left(f_{0}, f_{1}, \ldots, f_{m-1}\right) \cup e^{1} \cup C\left(f_{m+1}, \ldots\right)^{*} .
$$

The space $\operatorname{Im}\left(g_{m}\right) \subset C\left(f_{0}, f_{1}, \ldots, f_{m-1}\right) \cup e^{1}$ is contractible with respect to the point $e^{1} \cap C\left(f_{m+1}, \ldots\right)^{*}$; therefore we may assume that the mapping $f$ is homotopic, with respect to the subspace $C\left(f_{m+1}, \ldots\right)^{*}$, to the mapping $g_{m}$, the image of which lies in $C\left(f_{m+1}, \ldots\right)^{*}$.

Since the relative $C W$-complexes $\left(C\left(f_{k}, f_{k+1}, \ldots\right)^{*}, C\left(f_{k+1}, f_{k+2}, \ldots\right)^{*}\right)$ for $k>$ $m$ contain only one 1-dimensional cell plus only cells of the dimensions larger than $n$, it follows that $g_{m}$ (and therefore $f$ ) is homotopic relative to $p t$, to the constant mapping to the point $*$. Therefore $\pi_{n}\left(P^{*}, *\right)=0$.

The property of homological local connectedness with respect to singular homology HLC at all points, except at the base point, follows by the fact that the space $C\left(f_{0}, f_{1}, f_{2}, \ldots\right)$, being a $C W$-complex, is always locally contractible. As we have seen, it is easy to show that $\pi_{n}\left(C\left(f_{m}, f_{m+1}, f_{m+2}, \ldots\right)^{*}, *\right)=0$. It now follows by the Hurewicz Theorem that singular homology groups are trivial:

$$
H_{n}\left(C\left(f_{m}, f_{m+1}, f_{m+2}, \ldots\right)^{*}, *\right)=0, \text { for } n<m .
$$

Hence the space $P^{*}$ is also an HLC space at the base point. The clc property follows from HLC (cf. 44).

Finally, let us verify the acyclicity of $P^{*}$. Since $\pi_{n}\left(P^{*}, *\right)=0$ for all $n \geq 0$, it follows by the Hurewicz Theorem that the singular homology groups of $P^{*}$ are trivial for all $n, H_{n}\left(P^{*}, *\right)=0$. It is well-known that all classical homologies are naturally isomorphic on the category of compact metrizable HLC spaces (cf. e.g. [4). Therefore the space $P^{*}$ is acyclic in Čech, Borel-Moore, and Vietoris homology theories. By invoking the Universal Coefficients Theorem, we can conclude that over $\mathbb{Z}$, all singular, Čech, Alexander-Spanier, and sheaf cohomology groups of the space $P^{*}$ are trivial, too. 


\section{The infinite-Dimensional HaWAiIAN EARRINGS AND THE INFINITE-DIMENSIONAL HAWAIIAN GROUP}

The $n$-dimensional Hawaiian set of a space $X, n \in\{0,1,2, \ldots\}$, with the base point $x_{0} \in X$ is defined as the set of all homotopy classes $[f]$ of the mappings

$$
f:\left(\mathcal{H}^{n}, \theta\right) \rightarrow\left(X, x_{0}\right)
$$

of the $n$-dimensional Hawaiian earrings $\mathcal{H}^{n}$ into $X$. For $n \geq 1$ there is a natural multiplication with respect to which this set is a group. We denote it by $\mathcal{H}_{n}\left(X, x_{0}\right)$ and call it the Hawaiian group in dimension $n$ (cf. [8]).

The Hawaiian groups $\mathcal{H}_{n}\left(X, x_{0}\right)$ (the set $\mathcal{H}_{0}\left(X, x_{0}\right)$ ) are homotopy invariant in the category of all topological spaces with base points and continuous mappings. Note that for the cone over the 1-dimensional Hawaiian earrings the group $\mathcal{H}_{1}\left(C\left(\mathcal{H}^{1}\right), p t\right)$ is nontrivial, for some points $p t$ (cf. [8]).

The space $P^{*}$ is locally contractible at all points except the base point, and for every natural number $n$ there exists a neighborhood $U_{*}$ such that $\pi_{n}\left(U_{*}\right)$ is trivial. Therefore (since $\pi_{n}\left(P^{*}\right)=0$ ) it follows that $\mathcal{H}_{n}\left(P^{*}\right)=0$.

Consider a compact bouquet of Hawaiian earrings of all dimensions $\mathcal{H}^{\infty}=$ $\bigvee_{n=1}^{\infty} \mathcal{H}^{n}$ with respect to their base points. Call the space $\mathcal{H}$ the infinite-dimensional Hawaiian earrings. There is a natural base point $p t$. We shall call the set of all homotopy classes of maps $\left[\left(\mathcal{H}^{\infty}, p t\right),\left(X, x_{0}\right)\right]$ with the natural multiplication the infinite-dimensional Hawaiian group $\mathcal{H}_{\infty}\left(X, x_{0}\right)$, where $x_{0}$ is the base point of the space $X$.

Theorem 4.1. The infinite-dimensional Hawaiian group of the spaces $P^{*}$ constructed by the admissible spectra of Taylor is nontrivial, $\mathcal{H}_{\infty}\left(P^{*}, *\right) \neq 0$.

Proof. The inverse spectrum of Taylor can be described as follows. Let $M$ be the Moore space $=S^{2 q-1} \cup_{p} e^{2 q}, p \geq 3$. Toda bracket gives the mapping $f$ : $S^{2(p-1)}(M) \rightarrow M$ of the $2(p-1)$-fold suspension of $M$ to $M$. Let the space $P_{1}$ be the singleton $\left\{p_{1}\right\}, P_{2}=M, P_{i+2}=S^{2(p-1) i}(M)$ and $f_{2}=f, f_{i+1}=S^{2(p-1)}\left(f_{i}\right)$. Then we get the desired inverse spectrum.

According to Adams and Toda we have mappings $\phi$ and $\psi_{i}$ such that the composition $\phi f_{2} f_{3} \cdots f_{i} \psi_{i}$ is a nontrivial Toda's element $\alpha_{i}$ (cf. [1, pp. 12-13], [14]):

$$
\begin{array}{rll}
P_{1} \stackrel{f_{1}}{\leftarrow} P_{2} & \stackrel{f_{2}}{\leftarrow} P_{3} \stackrel{f_{3}}{\leftarrow} \ldots \stackrel{f_{i+1}}{\leftarrow} & P_{i+2} \leftarrow \cdots \\
\varphi \downarrow & & \uparrow \psi_{i} \\
S^{2 q} & & S^{2 q-1+2(p-1) i}
\end{array}
$$

Define the mapping $f: \mathcal{H}^{\infty} \rightarrow P^{*}$ as follows. Consider the compact bouquet of spheres $\bigvee_{i=1}^{\infty} S^{2 q-1+2(p-1) i}$. On every sphere $S^{2 q-1+2(p-1) i}$ we have a mapping $\psi_{i}$ to $P_{i+2}$. The set of all mappings $\left\{\psi_{i}\right\}$ naturally generates the mapping of $\bigvee_{i=1}^{\infty} S^{2 q-1+2(p-1) i}$ to $P^{*}$. The space $\bigvee_{i=1}^{\infty} S^{2 q-1+2(p-1) i}$ can be considered as a subspace of $\mathcal{H}^{\infty}$.

Now let $f$ be the extension of this mapping to the entire space $\mathcal{H}^{\infty}$ which maps the complement to $*$. The mapping $f$ is an essential mapping.

Indeed, suppose that $f$ were inessential. Then we would have a homotopy $H$ : $\mathcal{H}^{\infty} \times[0,1] \rightarrow P^{*}$ such that $H(\theta, 0)=*$. The restrictions of $H$ on every sphere $S^{2 q-1+2(p-1) i}$ would be inessential in the space $P^{*} \backslash\left\{p_{1}\right\}$ for large $i$. 
For simplicity we shall again denote these restrictions by $H$. So we have for a large $i$ the homotopy

$$
H: S^{2 q-1+2(p-1) i} \times[0,1] \rightarrow P^{*},
$$

connecting the mapping $\psi_{i}$ and the constant mapping in $P^{*} \backslash\left\{p_{1}\right\}$. The $C W$ complex $S^{2 q-1+2(p-1) i} \times[0,1]$ is a $(2 q+2(p-1) i)$-dimensional complex.

Choose an integer $m>2 q+2(p-1) i$ and consider the relative $C W$-complex $\left(P^{*}, C\left(f_{m+1}, f_{m+2}, \cdots\right)^{*}\right)$. By the Cellular Approximation Theorem the mapping

$$
H: S^{2 q-1+2(p-1) i} \times[0,1] \rightarrow P^{*} \backslash\left\{p_{1}\right\}
$$

is homotopy equivalent to a cellular map with respect to the set $S^{2 q-1+2(p-1) i} \times\{1\}$.

Since the complex $C\left(f_{m+1}\right)$ contains only two 0-cells, one 1-cell, plus cells of dimension larger than $2 q+2(p-1) i$, we may assume that the image of the homotopy

$$
H: S^{2 q-1+2(p-1) i} \times[0,1] \rightarrow P^{*} \backslash\left\{p_{1}\right\}
$$

lies in the space

$$
C\left(f_{1}, \ldots, f_{m-1}\right) \cup e^{1} \cup C\left(f_{m+1}, \ldots\right)^{*} \backslash\left\{p_{1}\right\} .
$$

Now, the space $P_{2}$ is a retract of this space. So we have a mapping of the sphere $S^{2 q-1+2(p-1) i}$ to the sphere $S^{2 q}$, which should be inessential. But this contradicts the nontriviality of the Toda element mentioned above. Therefore $\mathcal{H}_{\infty}\left(P^{*}, *\right) \neq$ 0 .

\section{EPIlogue}

Since our example in [8] is infinite-dimensional, it is natural to ask the following question [5]:

Problem 5.1. Does there exist a finite-dimensional noncontractible Peano continuum all homotopy groups of which are trivial?

Our Theorem 3.1 gives an answer to our problem from 8 but the cases of finitedimensional spaces and infinite-dimensional Hawaiian groups remain open:

Problem 5.2. Let $P$ (resp. $P^{*}$ ) be the one-point compactification of any finitedimensional countable polyhedron by the point $\theta \in P$ (resp. $\left.\theta^{*} \in P^{*}\right)$. Suppose that $f:(P, \theta) \rightarrow\left(P^{*}, \theta^{*}\right)$ is any continuous mapping such that

$$
\mathcal{H}_{n}(f): \mathcal{H}_{n}(P, \theta) \rightarrow \mathcal{H}_{n}\left(P^{*}, \theta^{*}\right)
$$

is an isomorphism for every $n \in \mathbb{N}$. Is $f$ then a homotopy equivalence?

Problem 5.3. Let $P$ (resp. $\left.P^{*}\right)$ be the one-point compactification of a connected polyhedron by the point $\theta \in P\left(\right.$ resp. $\left.\theta^{*} \in P^{*}\right)$. Suppose that $f:(P, \theta) \rightarrow\left(P^{*}, \theta^{*}\right)$ is a continuous mapping such that

$$
\mathcal{H}_{\infty}(f): \mathcal{H}_{\infty}(P, \theta) \rightarrow \mathcal{H}_{\infty}\left(P^{*}, \theta^{*}\right)
$$

is an isomorphism. Is $f$ then a homotopy equivalence?

\section{ACKNOWLEDGEMENTS}

This research was supported by the Slovenian Research Agency grants P1-02920101, J1-9643-0101 and J1-2057-0101. We thank the referee for comments and suggestions. 


\section{REFERENCES}

[1] J. F. Adams, Talk on Toda's work, in: Homotopy Theory and Related Topics, Lect. Notes Math. 1418, Springer-Verlag, Berlin, 1990, pp. 7-14. MR.1048172 (91c:55022)

[2] J. F. Adams, On the groups $J(X)$. IV, Topology 5 (1966), 21-71. MR0198470 (33:6628)

[3] K. Borsuk, Theory of Retracts, Monogr. Mat. 44, PWN, Warsaw, 1967. MR0216473 (35:7306)

[4] G. E. Bredon, Sheaf Theory, 2nd ed., Graduate Texts Math., 170, Springer-Verlag, Berlin, 1997. MR1481706 (98g:55005)

[5] K. Eda, U. H. Karimov, D. Repovš, A nonaspherical cell-like 2-dimensional simply connected continuum and related constructions, Topology Appl. 156 (2009), 515-521. MR2492298

[6] A. Hatcher, Vector Bundles and K-Theory, lecture notes, Cornell University, Ithaca, NY, http://www.math.cornell.edu/ hatcher/VBKT/VBpage.html.

[7] D. S. Kahn, An example in Čech cohomology, Proc. Amer. Math. Soc. 16 (1965), 584. MR0179785 (31:4027)

[8] U. H. Karimov, D. Repovš, Hawaiian groups of topological spaces, Uspehi. Math. Nauk. 61:5 (2007), 185-186. (in Russian); English transl. in Russian Math. Surv. 61:5 (2006), 987-989. MR2328264 (2008d:55009)

[9] M. Karoubi, K-Theory, Springer-Verlag, Berlin, 1978. MR.0488029 (58:7605)

[10] J. Krasinkiewicz, On a method of constructing ANR-sets. An application of inverse limits, Fund. Math. 92 (1976), 95-112. MR0420546 (54:8560)

[11] S. B. Nadler, Jr., Continuum Theory. An Introduction, Monogr. and Textbooks in Pure and Appl. Math., 158, Marcel Dekker, Inc., New York, 1992. MR1192552 (93m:54002)

[12] L. C. Siebenmann, Chapman's classification of shapes: a proof using collapsing, Manuscr. Math. 16 (1975), 373-384. MR0431183 (55:4185)

[13] J. L. Taylor, A counterexample in shape theory, Bull. Amer. Math. Soc. 81 (1975), 629-632. MR0375328(51:11523)

[14] H. Toda, On unstable homotopy of spheres and classical groups, Proc. Nat. Acad. Sci. U.S.A. 46 (1960), 1102-1105. MR0123329 (23:A657)

[15] G. W. Whitehead, Elements of Homotopy Theory, Springer-Verlag, Berlin, 1978. MR516508 (80b:55001)

Institute of Mathematics, Academy of Sciences of Tajikistan, Ul. Ainy 299A, Dushanbe 734063, TAJIKISTAN

E-mail address: umedkarimov@gmail.com

Faculty of Mathematics and Physics, and Faculty of Education, University of Ljubljana, P.O. Box 2964, Luubluana 1001, Slovenia

E-mail address: dusan.repovs@guest.arnes.si 